\title{
A HIGHER DIMENSION GENERALIZATION OF THE SINE-GORDON EQUATION AND ITS BÄCKLUND TRANSFORMATION
}

\author{
BY KETI TENENBLAT ${ }^{1}$ AND CHUU-LIAN TERNG ${ }^{2}$
}

The classical Bäcklund theorem ([1], [4], [5]) studies the transformation of hyperbolic (i.e. constant negative curvature) surfaces in $R^{3}$ by realizing them as focal surfaces of pseudo-spherical line congruences. The integrability theorem says that one can construct a family of new hyperbolic surfaces in $R^{3}$ from a given one. Bianchi showed how to construct algebraically another family of hyperbolic surfaces from this family.

It is well known that there is a correspondence betwen solutions of the Sine-Gordon equation

SGE

$$
\frac{\partial^{2} \phi}{\partial x^{2}}-\frac{\partial^{2} \phi}{\partial t^{2}}=\sin \phi
$$

and hyperbolic surfaces in $R^{3}$ ([1], [4], [5]). Therefore Bäcklund's theorem provides a method for generating new solutions of SGE from a given one, and Bianchi's permutability theorem [5] enables one to construct more solutions by an algebraic formula. This technique has recently received much attention in the studies of soliton solutions of SGE [2] and has been used successfully in the study of solitons of other nonlinear equations of evolution in one space dimension. But generalizations to more space variables has been less successful.

A natural generalization would be to find a transformation theory for hyperbolic (i.e. constant negative sectional curvature) submanifolds in Euclidean space. É. Cartan [3] showed that hyperbolic $n$-manifolds locally immerse in $R^{2 n-1}$, but not in $R^{2 n-2}$. Moreover, [3] he proved the existence of "line of curvature coordinates", in which all components of the second fundamental form are diagonalized. J. D. Moore [6] improved this result and we have:

Theorem 1 (É. CARTAN). Suppose $M$ is a hyperbolic $n$-submanifold of $R^{2 n-1}$. Then locally $M$ can be parametrized by its lines of curvature so that

Received by the editors December 4, 1978.

AMS (MOS) subject classifications (1970). Primary 53B25; Secondary 35L60.

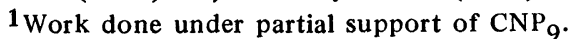

2 Work done under partial support of NSF grant MCS 76-01692. 


$$
\begin{aligned}
\mathrm{I} & =\sum_{i=1}^{n}\left(a_{i}\right)^{2} d u_{i}^{2}, \\
\mathrm{II} & =\sum_{i=1}^{n} \sum_{m=n+1}^{2 n-1} b_{i m}\left(a_{i}\right)^{2} d u_{i}^{2} e_{m},
\end{aligned}
$$

where $\sum_{i=1}^{n}\left(a_{i}\right)^{2}=1$ and $e_{n+1}, \ldots, e_{2 n-1}$ is an orthonormal local frame field for the normal bundle of $M$. In particular, the normal bundle is flat. Moreover, $\sum_{i=1}^{n} \partial / \partial u_{i}$ is the unique unit asymptotic vector in the first orthant.

We call such coordinates "generalized Tchebyshef coordinates".

Definition 1 . Let $E_{1}$ and $E_{2}$ be two $k$-planes in a $2 k$-dimensional inner product space $(V,\langle\rangle)$ and $P: V \rightarrow E_{1}$ the orthogonal projection. Define a symmetric bilinear form on $E_{2}$ by $\left(v_{1}, v_{2}\right)=\left\langle P\left(v_{1}\right), P\left(v_{2}\right)\right\rangle$. The $k$ angles between $E_{1}$ and $E_{2}$ are defined to be $\theta_{1}, \ldots, \theta_{k}$, where $\cos ^{2} \theta_{1}, \ldots, \cos ^{2} \theta_{k}$ are the $k$ eigenvalues for the selfadjoint operator $A: E_{2} \rightarrow E_{2}$ such that $\left(v_{1}, v_{2}\right)=\left\langle A v_{1}, v_{2}\right\rangle$.

Definition 2. A line congruence between two $n$-submanifolds $M$ and $M^{*}$ in $R^{2 n-1}$ is a diffeomorphism $l: M \rightarrow M^{*}$ such that for $P \in M$ the line joining $P$ and $P^{*}=l(P)$ is a common tangent line for $M$ and $M^{*}$.

For a line congruence $l: M \rightarrow M^{*}$ between two $n$-submanifolds in $R^{2 n-1}$, the normal planes $\nu_{P}$ and $\nu_{P^{*}}^{*}$ at corresponding points $P$ and $P^{*}$ are of dimension $n-1$ and are perpendicular to $\overrightarrow{P P *}$. Therefore they lie in a common $2 n-2$ dimensional inner product space, so there are $n-1$ angles between them.

Definition 3. A line congruence $l: M \rightarrow M^{*}$ between two $n$-submanifolds in $R^{2 n-1}$ is called pseudo-spherical (p.s.) if

(1) The distance (between $P$ and $P^{*}$ ) is a constant $r$, independent of $P$.

(2) The $n-1$ angles between $\nu_{P}$ and $\nu_{P *}^{*}$ are the same and equal to a constant $\theta$, independent of $P$.

(3) The normal bundles $\nu$ and $\nu^{*}$ are flat.

(4) The bundle map $\Gamma: \nu \rightarrow \nu^{*}$ given by the orthogonal projection commutes with the normal connections.

Then we have the following generalization of Bäcklund's theorem.

TheOREM 2. Suppose there is a p.s. congruence l: $M \rightarrow M^{*}$ of $n$-submanifolds in $R^{2 n-1}$ with distance $r$ and angle $\theta$. Then both $M$ and $M^{*}$ have constant sectional curvature $-((\sin \theta) / r)^{2}$.

TheOREM 3. Suppose $M$ is a hyperbolic n-submanifold in $R^{2 n-1}$ with sectional curvature $K=-((\sin \theta) / r)^{2}$, where $r$ and $\theta$ are constants. Let $v_{1}^{0}, \ldots$, $v_{n}^{0}$ be the orthonormal base at $P_{0}$ consisting of principal curvature vectors, and $v_{0}=\sum_{i=1}^{n} c_{i} v_{i}^{0}$ a unit vector with $c_{i} \neq 0$ for $1 \leqslant i \leqslant n$. Then there exists a local $n$-submanifold $M^{*}$ of $R^{2 n-1}$ and a p.s. congruence $l: M \rightarrow M^{*}$ such that if $P_{0}^{*}=l\left(P_{0}\right)$ we have ${\overrightarrow{P_{0}}}_{0}^{*}=r v_{0}$ and $\theta$ is the angle between the normal planes at $P_{0}$ and $P_{0}^{*}$. 
The above results are joint work of both authors; the following results were obtained by the second author.

THEOREM 4. Suppose $l: M \rightarrow M^{*}$ is a p.s. congruence of hyperbolic $n$-submanifolds in $R^{2 n-1}$. Then the generalized Tchebyshef coordinates (hence lines of curvature and asymptotic curves) correspond under $l$.

Bianchi's permutability theorem generalizes to

THEOREM 5. Let $l_{1}: M_{0}^{n} \rightarrow M_{1}^{n}, l_{2}: M_{0}^{n} \rightarrow M_{2}^{n}$ be two p.s. congruences in $R^{2 n-1}$ with angles $\theta_{1}, \theta_{2}$ respectively. If $\theta_{1} \neq \theta_{2}$, then there exists a unique hyperbolic $n$-submanifold $M_{3}$ of $R^{2 n-1}$ and p.s. congruences $\bar{l}_{1}: M_{1} \rightarrow M_{3}$, $\bar{l}_{2}: M_{2} \rightarrow M_{3}$ with angles $\theta_{2}, \theta_{1}$ respectively.

For the analytic part of this theory, one needs to find the appropriate partial differential equations.

In what follows $M$ will be a hyperbolic $n$-submanifold (with curvature -1) in $R^{2 n-1}$, and $\left(u_{1}, \ldots, u_{n}\right)$ etc. as in Theorem 1. Associate to $M$ a map $A=\left(a_{i j}\right): R^{n} \rightarrow O(n)$ defined by

$$
\begin{gathered}
a_{1 j}=a_{j}, \quad 1 \leqslant j \leqslant n, \\
a_{i j}=b_{j}^{n+i-1} a_{j}, \quad 1 \leqslant j \leqslant n, 2 \leqslant i \leqslant n .
\end{gathered}
$$

Then $A$ satisfies the following second order system given by the Gauss and Codazzi equations:

$$
\begin{array}{r}
\frac{\partial}{\partial u_{j}}\left(\frac{1}{a_{1 j}} \frac{\partial a_{1 j}}{\partial u_{j}}\right)+\frac{\partial}{\partial u_{i}}\left(\frac{1}{a_{1 i}} \frac{\partial a_{1 j}}{\partial u_{i}}\right)+\sum_{k \neq i, j} \frac{1}{a_{1 k}^{2}} \frac{\partial a_{1 i}}{\partial u_{k}} \frac{\partial a_{1 j}}{\partial u_{k}}=a_{1 i} a_{1 j}, \\
\text { GSGE } \frac{\partial}{\partial u_{k}}\left(\frac{1}{a_{1 j}} \frac{\partial a_{1 i}}{\partial u_{j}}\right)=\frac{1}{a_{1 k} a_{1 j}} \frac{\partial a_{1 i}}{\partial u_{k}} \frac{\partial a_{1 k}}{\partial u_{j}}, \quad i, j, k \text { distinct, }
\end{array}
$$$$
\frac{\partial a_{j k}}{\partial u_{i}}=\frac{a_{j i}}{a_{1 i}} \frac{\partial a_{1 k}}{\partial u_{i}}, \quad i, j, k \text { distinct. }
$$

Conversely, the complete integrability of the Gauss and Codazzi equations implies that there exists a hyperbolic $n$-submanifold of $R^{2 n-1}$ for a given solution of GSGE, so there is a correspondence between \{hyperbolic $n$-submanifolds of $\left.R^{2 n-1}\right\}$ and $\left\{A: R^{n} \rightarrow O(n)\right.$, solutions of GSGE $\}$, and the correspondence is unique up to a left-translation by a constant $O(n-1)$ matrix or a diagonal $O(n)$ matrix. For $n=2$, we have $A=\left(\begin{array}{c}\cos \phi \sin \phi \\ \sin \phi-\cos \phi\end{array}\right)$ and GSGE is $\partial^{2} \phi / \partial u_{1}^{2}-$ $\partial^{2} \phi / \partial u_{2}^{2}=\sin \phi \cos \phi$, hence GSGE is a generalization of SGE to higher dimensions. 
Let $l: M \rightarrow M^{*}$ be a p.s. congruence with angle $\theta, e_{1}, \ldots, e_{2 n-1}$ an orthonormal frame field on $M$ such that the induced normal connection with respect to normal frame $e_{n+1}, \ldots, e_{2 n-1}$ is zero, and $v_{1}, \ldots, v_{n}$ the orthonormal frame field on $M$ consisting of principal curvature vectors. Suppose $e_{i}=$ $\sum_{j=1}^{n} x_{i j} v_{j}$, for $1 \leqslant i \leqslant n$, then $X=\left(x_{i j}\right)$ is the corresponding $O(n)$-map for $M^{*}$. Hence one has the following analytic formulations of the geometric theorems:

Theorem 6. Let $A=\left(a_{i j}\right): R^{n} \rightarrow O(n)$ be a solution of GSGE. Then the following first order completely integrable system:

$$
(d X) X^{t}+X \Phi X^{t}=X \delta A^{t} D-D A \delta X^{t}
$$

gives a new solution for GSGE, where $\Phi=\left(\phi_{i j}\right)$ is the Levi-Civita connection 1-form on $M$, in fact $\phi_{i j}=1 / a_{1 i} \partial a_{1 j} / \partial u_{i} d u_{j}-1 / a_{1 j} \partial a_{1 i} / \partial u_{j} d u_{i}, \delta=$ $\operatorname{diag}\left(d u_{1}, \ldots, d u_{n}\right)$, and $D=\operatorname{diag}(\csc \theta, \cot \theta, \ldots, \cot \theta)$.

THEOREM 7. Suppose $A_{0}$ is a solution of GSGE and $A_{i}$ 's are solutions of GSGE obtained from $A_{0}$ by solving $B T\left(\theta_{i}\right)$ for $i=1,2$. Then a fourth solution $A_{3}$ can be obtained by the following algebraic formula:

$$
A_{3} A_{0}^{-1}=\left(-D_{2}+D_{1} A_{2} A_{1}^{-1}\right)\left(D_{1}-D_{2} A_{2} A_{1}^{-1}\right)^{-1} J
$$

where $D_{i}=\operatorname{diag}\left(\csc \theta_{i}, \cot \theta_{i}, \ldots, \cot \theta_{i}\right)$, and $J=\operatorname{diag}(-1,1, \ldots, 1)$.

REMARK 1.

$$
\left(\begin{array}{cc}
D_{1} & -D_{2} \\
-D_{2} & D_{1}
\end{array}\right)
$$

is an element in $O(n, n)$, a group which acts on $O(n)$ by linear fractional transformation, hence the right-hand side of (*) belongs to $O(n)$.

REMARK 2. For $n=2, B T(\theta)$ is the classical Backlund transformation for SGE:

$$
\begin{aligned}
& \frac{\partial \alpha}{\partial u_{1}}+\frac{\partial \phi}{\partial u_{2}}=\cot \theta \cos \alpha \sin \phi+\csc \theta \sin \alpha \cos \phi \\
& \frac{\partial \alpha}{\partial u_{2}}-\frac{\partial \phi}{\partial u_{1}}=-\cot \theta \sin \alpha \cos \phi-\csc \theta \cos \alpha \sin \phi
\end{aligned}
$$

where

$$
A=\left(\begin{array}{rr}
\cos \phi & \sin \phi \\
\sin \phi & -\cos \phi
\end{array}\right), X=\left(\begin{array}{cr}
\cos \alpha & \cos \alpha \\
\sin \alpha & -\cos \alpha
\end{array}\right)
$$

The formula (*) above is called Bianchi's superposition formula by physists, and becomes 
where

$$
\tan \frac{\phi_{3}-\phi_{0}}{2}=\frac{\cos \theta_{2}-\cos \theta_{1}}{\cos \left(\theta_{2}-\theta_{1}\right)^{-1}} \tan \frac{\phi_{2}-\phi_{1}}{2}
$$

$$
A_{i}=\left(\begin{array}{cc}
\cos \phi_{i} & \sin \phi_{i} \\
\sin \phi_{i} & -\cos \phi_{i}
\end{array}\right)
$$

If $A$ is taken to be the identity everywhere one gets a trivial ("vacuum") solution of GSGE. Applying $B T(\theta)$ with varing initial conditions to this solution gives families of solutions including the one dimensional solitary wave solutions of GSE. Finally, applying the superposition formula (*) consecutively to these families gives further families of solutions which generalize the $n$-solition solutions of SGE. A fuller discussion of these solutions will appear elsewhere together with a proof of the above theorems.

The authors would like to thank Professor S. S. Chern for many helpful suggestions.

\section{REFERENCES}

1. A. V. Bäcklund, Concerning surfaces with constant negative curvatures, translated by E. M. Coddington, Lancaster, Pa. New Era Printing Co.

2. A. Barone, F. Esposito, C. J. Magee and A. C. Scott, Theory and applications of the Sine-Gordon equation, Riv. Nuovo Cimento 1 (1971), 227-267.

3. '́. Cartan, Sur les variétés de courbure constante d'un space euclidien ou noneuclidien, Bull. Soc. Math. France 47 (1919), 125-160; 48 (1920), 132-208.

4. S. S. Chern and C. L. Terng, An analogue of Bäcklund's theorem in affine geometry, Rocky Mountain J. (to appear).

5. L. P. Eisenhart, A treatise in the differential geometry of curves and surfaces, Ginn and Company, New York, 1909.

6. J. D. Moore, Isometric immersions of space forms in space forms, Pacific J. Math. 40 (1972), 157-166.

DEPARTMENT OF MATHEMATICS, UNIVERSITY OF CALIFORNIA, BERKELEY, CALIFORNIA 94720 BRAZIL

DEPARTMENT OF MATHEMATICS, UNIVERSITY OF BRASILIS, BRASILIA,

DEPARTMENT OF MATHEMATICS, PRINCETON UNIVERSITY, PRINCETON, NEW JERSEY 08540 
